\title{
Migrating lumbar intrathecal catheter fragment associated with intracranial subarachnoid hemorrhage
}

\author{
Luke Hnenny, MD, Hatem A. Sabry, MD, Jeffrey S. Raskin, MS, MD, Jesse J. Liu, MD, \\ Neil E. Roundy, MD, and Aclan Dogan, MD \\ Department of Neurological Surgery, Oregon Health \& Science University, Portland, Oregon
}

\begin{abstract}
Intrathecal catheter placement into the lumbar cistern has varied indications, including drug delivery and CSF diversion. These Silastic catheters are elastic and durable; however, catheter-associated malfunctions are well reported in the literature. Fractured catheters are managed with some variability, but entirely intradural retained fragments are often managed conservatively with observation.

The authors describe a case of a 70-year-old man with an implanted intrathecal morphine pump for failed back surgery syndrome who presented to an outside hospital with a history of headache, neck pain, nausea, and photophobia of 3 days' duration. He also described mild weakness and intermittent numbness of both legs. Unenhanced head CT demonstrated subarachnoid hemorrhage (SAH). A right C-5 hemilaminectomy was performed.

This case is unique in that there was no indication that the lumbar intrathecal catheter had fractured prior to the patient's presentation with $\mathrm{SAH}$. This case demonstrates that intrathecal catheter fragments are mobile and can precipitate intracranial morbidity. Extrication of known fragments is safe and should be attempted to prevent further neurosurgical morbidity.
\end{abstract}

http://thejns.org/doi/abs/10.3171/2014.9.SPINE147

KEY WORDS intrathecal morphine pump; subarachnoid hemorrhage; intracranial morbidity; vascular disorders; lumbar spine

\section{$\mathrm{L}$}

UMBAR intrathecal catheters are placed commonly by neurosurgeons and are used for numerous purposes. These include delivery of intrathecal narcotic analgesics and muscle relaxants, perioperative drainage of CSF to facilitate brain relaxation during intracranial surgery, perioperative drainage of CSF to facilitate spinal cord perfusion during open or endovascular repair of thoracoabdominal aortic aneurysms ${ }^{4}$ intermittent drainage of CSF for diagnosis of normal-pressure hydrocephalus, and drainage of CSF to facilitate resolution of a CSF fistula.

When used for administration of intrathecal medications, lumbar intrathecal catheters are inserted into the spinal subarachnoid space, tunneled subcutaneously, and connected to a mechanical pump anchored to the abdominal fascia. Infusion rate adjustments and refilling of the pump are performed in a percutaneous fashion. Devicerelated complications associated with these drug infusion systems are not uncommon.

We report the unusual case of a patient presenting with posterior fossa subarachnoid hemorrhage (SAH) associated with fracture and migration of a narcotic pump lumbar intrathecal catheter into the upper cervical subarachnoid space and infratentorial cisterns.

\section{Case Report}

Clinical Presentation

A 70-year-old man with an implanted intrathecal morphine pump for failed back surgery syndrome presented to an outside hospital with a history of headache, neck pain, nausea, and photophobia of 3 days' duration. He also described mild weakness and intermittent numbness of both legs. An unenhanced head CT scan demonstrated $\mathrm{SAH}$ in the infratentorial cisterns, most prominently in the prepontine cistern. Surprisingly, a migrated intrathecal catheter with the tip in the prepontine area, wrapping inferiorly and posterolaterally down through the foramen magnum and upper cervical subarachnoid space, was also observed (Fig. 1). The caudal-most portion of the migrated 


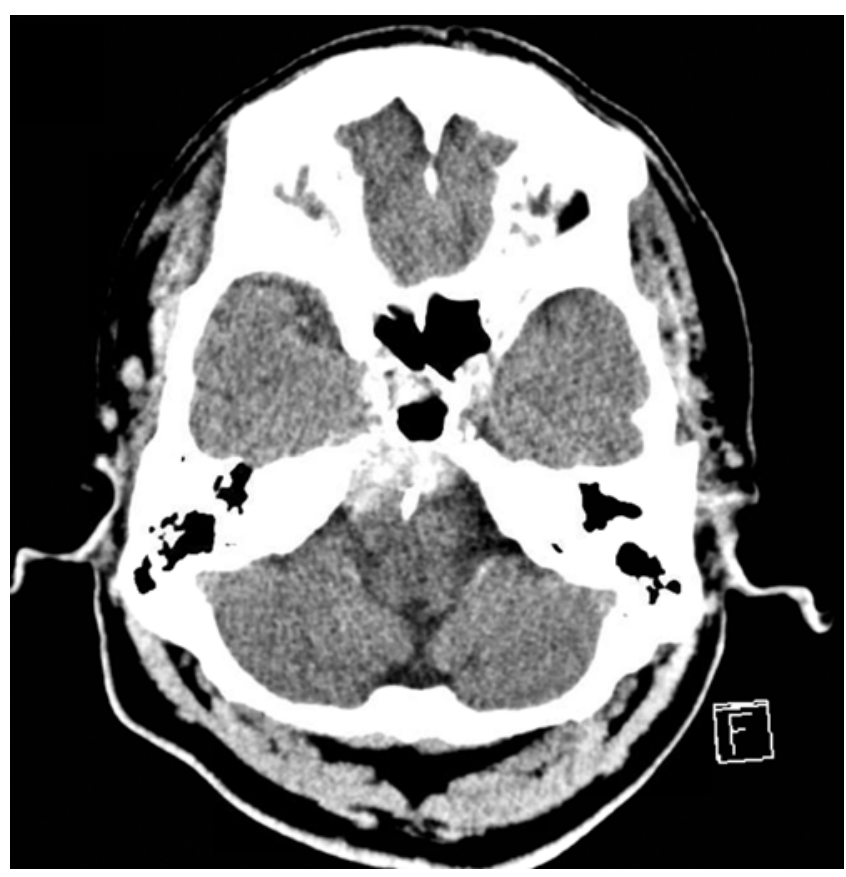

FIG. 1. Unenhanced head CT scan showing SAH in the prepontine cistern and associated catheter tip.

catheter was positioned posterolaterally on the right, at the C-5 level (Fig. 2). Based on these findings, the patient was transferred to our institution for care.

On admission, the results of neurological examination were normal. The patient's preexisting medical conditions included atrial fibrillation, Type 2 diabetes mellitus, hypertension, and an elevated cholesterol level. Medications included rivaroxaban, fenofibrate, atorvastatin, lisinopril, metoprolol, and hydrocodone-acetaminophen, as well as acetylsalicylic acid as needed for the most recent headache.

His coagulopathy was reversed immediately with prothrombin complex concentrate, activated factor VII, and platelets. Additional imaging was also performed. A 3D lumbar spine $\mathrm{CT}$ reconstruction showed a catheter con- nected to a pump subcutaneously, with premature termination of the catheter evident at the interspinous space of L2-3, where it had presumably sheared off (Fig. 3). A 3D reconstructed head CT angiogram showed the position of the migrated catheter in greater detail as well as its relationship to the vertebrobasilar circulation (Fig. 4). A conventional cerebral angiogram definitively ruled out a vascular lesion that could have been responsible for the SAH. No lesion was present.

Despite the fact that the patient was no longer receiving intrathecal morphine, he exhibited no signs of opiate withdrawal. The pain service was consulted, and his treatment was supplemented with oral and intravenous narcotics. No plans were made to remove the pump or proximal catheter during this hospitalization.

\section{Operation}

Of primary concern was that, if left in situ, the migrated catheter had the potential to cause repeated hemorrhage. After the risks and benefits of pump explantation were discussed with the patient, a right C-5 hemilaminectomy was performed. The dura was opened, and traction sutures were placed. The migrated catheter was easily visualized, resting posterolateral to the spinal cord. The catheter was gently pulled out with negligible resistance. No bleeding was encountered. The subarachnoid space was irrigated, and the dura was closed in a watertight fashion. The fascia, subcutaneous tissue, and skin were closed in the usual fashion. Postoperatively, the patient recovered and was discharged to home (out of state).

\section{Follow-Up}

At an 8-month postoperative follow-up telephone interview, the patient reported that at 5 months postoperatively he was not tolerating oral analgesics and he had elected to have a new morphine pump implanted. The patient reported that he is doing well, with no SAH or explantation surgery sequelae, his back pain is well controlled with the new morphine pump, and he is able to fully conduct activities of daily living.
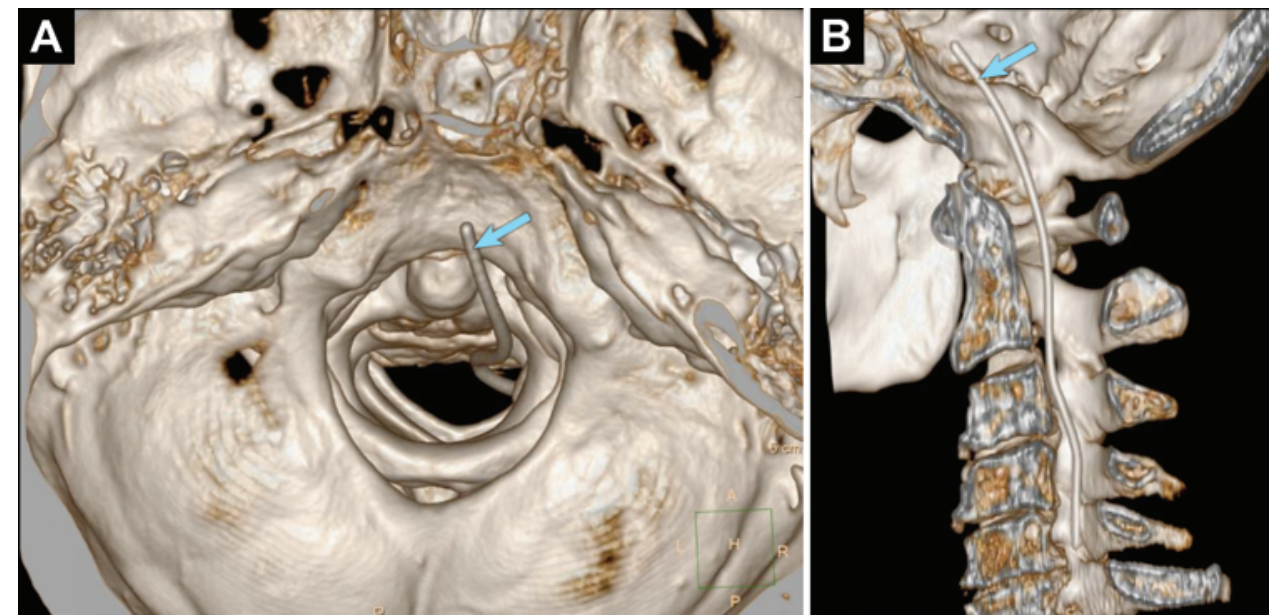

FIG. 2. 3D head (A) and upper cervical spine (B) CT reconstructions showing the location of the migrated catheter (arrows). Figure is available in color online only. 


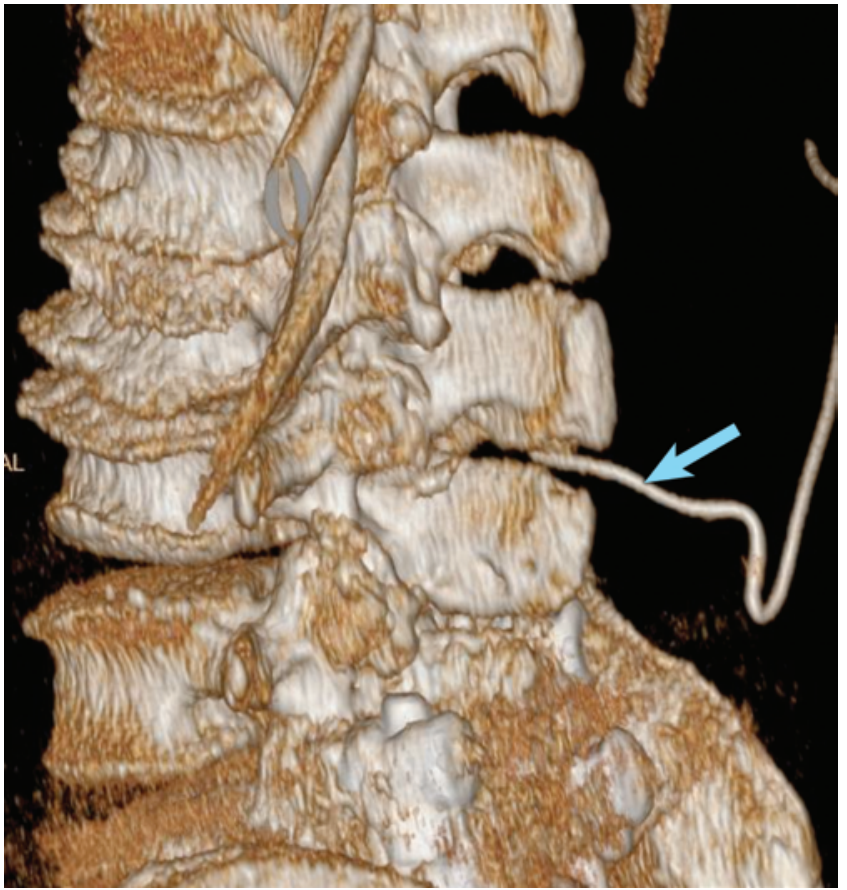

FIG. 3. 3D lumbar spine CT reconstruction showing the proximal portion of the catheter (arrow) terminating at the L2-3 interspinous space. Figure is available in color online only.

\section{Discussion}

Catheter-related complications are a known risk of the implantation of lumbar intrathecal catheter infusion systems and constitute $65 \%$ of device-related complications. ${ }^{5}$ The most common catheter-related complications are catheter leakage, dislocation of the catheter tip, disconnection at the pump, catheter occlusion, and granuloma formation at the catheter tip. ${ }^{5}$ In a prospective study involving 209 patients undergoing implantation of lumbar intrathecal catheter drug delivery systems for pain or spasticity, 7 (3.3\%) catheter-related complications were reported. ${ }^{6}$ Three patients $(1.4 \%)$ had leakage, cuts, or breaks in the catheter, and only 2 patients $(0.9 \%)$ had catheter dislodgement or migration. ${ }^{6}$

Numerous cases of migration of lumbar intrathecal catheters to unusual locations have been reported, including migration to the spinal cord, ${ }^{1}$ the neural foramen, ${ }^{10}$ and most significantly, the intracranial space. . $^{2,3,7,9,11,12}$ Lumboperitoneal shunt catheters are the most commonly reported culprits, since they are not affixed to a pump and, until recently, tended to be valveless. Migration of pump-associated catheters requires either disconnection from the pump or fracture of the catheter.

\section{Literature Review}

To our knowledge, there are 6 reported cases of intracranial migration of lumbar intrathecal catheters in the literature. Three cases involve valveless lumboperitoneal shunts, ${ }^{2,3,9}$ which are becoming less common. The remaining 3 cases are described below.

Nakaji et al. ${ }^{12}$ describe a patient with a baclofen pump and known catheter fracture at the L4-5 interspinous space. The pump and proximal catheter were explanted, and the fractured fragment was left in the thecal sac. The patient presented 10 days following explantation with $\mathrm{SAH}$ in the basal cisterns, and imaging showed the tip of the catheter lying adjacent to the basilar artery at the level of the superior cerebellar artery. The patient initially declined explantation of the migrated catheter; however,

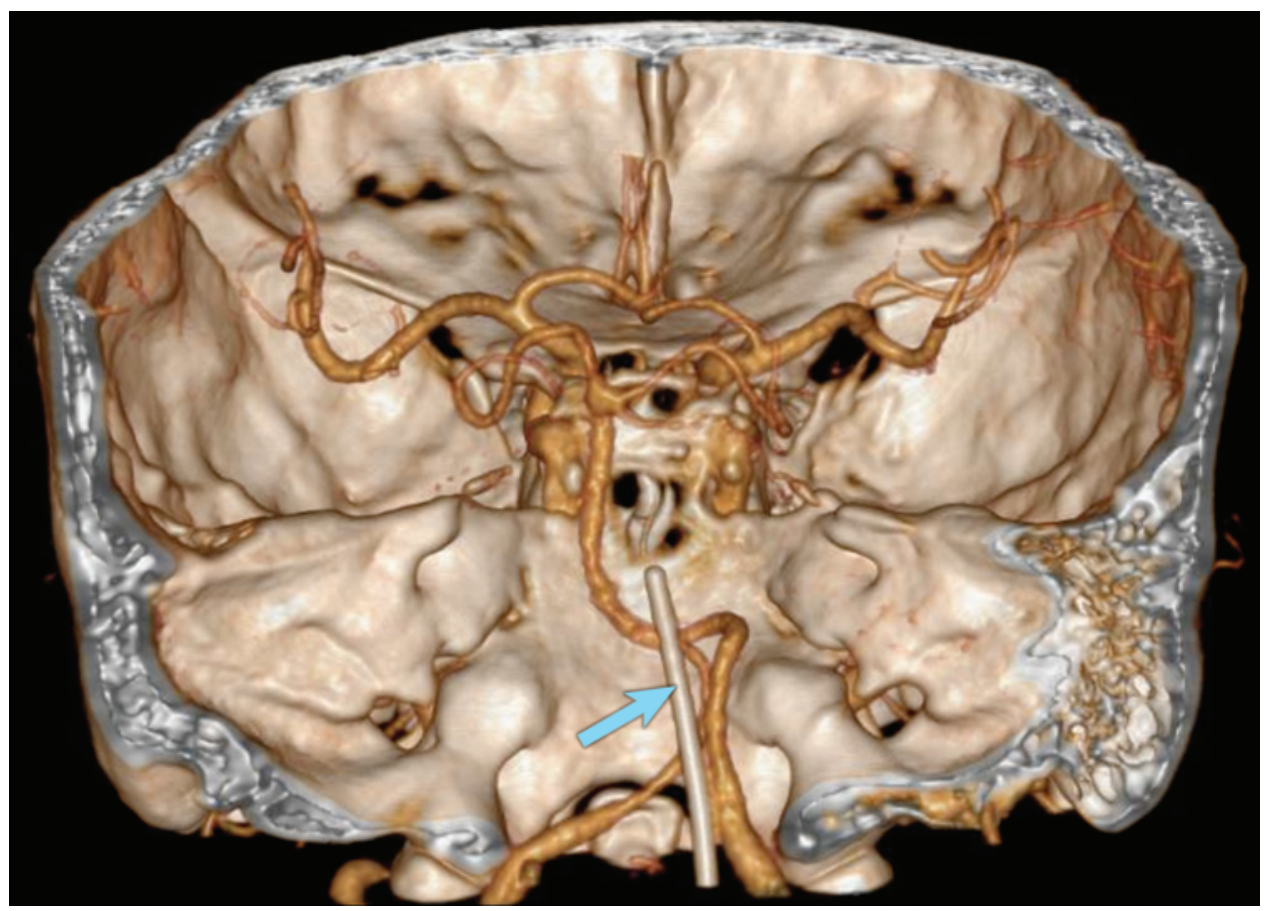

FIG. 4. 3D head CT angiogram reconstruction showing the relationship of the migrated catheter (arrow) to the vertebrobasilar circulation. Figure is available in color online only. 
5 months after the SAH, he elected to undergo removal. The removal was performed via a C6-7 laminoplasty and was uncomplicated.

Maugans ${ }^{11}$ reports a case of a 16-year-old male with a nonfunctioning baclofen pump who presented with back pain. Plain radiographs of the lumbar spine failed to show a catheter within the spinal canal, and it was assumed that the catheter had withdrawn into the abdominal and lumbar soft tissues. Subsequently, the patient presented with new neck pain, dizziness, and vomiting. Imaging revealed a migrated intrathecal catheter traversing the obex, fourth ventricle, and aqueduct, with its tip resting in the third ventricle. The patient underwent removal via the posterior atlanto-occipital membrane without complication.

Guppy and colleagues ${ }^{7}$ report a case of a 65 -year-old man who had undergone endovascular repair of a thoracic aortic aneurysm with adjunctive CSF drainage by means of a lumbar drain. Upon attempted removal of the lumbar drainage catheter, a large fragment of catheter sheared off and was retained; serial imaging followed. The patient presented subsequently with SAH in the basal cisterns. Imaging revealed a migrated intrathecal catheter with its distal end lying at the tip of the clivus and its proximal end situated lateral to the spinal cord at the T-3 level. The catheter was removed through a C3-4 laminectomy without complication.

Several theories regarding the mechanism of catheter migration into the intracranial space have been postulated. The most convincing explanation is that CSF pulsations related to the cardiac cycle cause net movement of the catheter in a cranial direction. This was postulated by Nakaji et al. ${ }^{12}$ as well as Maugans, ${ }^{11}$ based on the work of Henry-Feugeas et al. ${ }^{8}$ His group used phase-contrast cine MRI to study CSF flow intracranially and in the cervical spine. They reported that in the ventral subarachnoid space, CSF pulsations were caudad in systole and craniad in diastole. The net flow per cardiac cycle was craniad. Conversely, in the dorsal cervical subarachnoid space, flow was weaker than in the ventral, and indeed sometimes absent. When present, although systolic flow was caudad and diastolic flow was craniad as in the ventral cervical subarachnoid space, net flow was in a cranial direction. This net craniad flow in the dorsal subarachnoid space may contribute to upward migration of freefloating intrathecal catheters. Buoyancy was negated as a cause of craniad migration by Maugans, ${ }^{11}$ who reported that catheters of various lengths failed to float in a thecal sac model.

The mechanism of vascular injury in the case presented here is most likely venous, although this cannot be definitively proven. No arterial lesion could be demonstrated on angiography, and the clinical effects of the hemorrhage were milder than would be expected from an arterial injury. We suspect that the catheter tip disrupted small veins in the prepontine cistern, causing SAH. In cases such as ours, the catheter tip could become dangerous when subjected to the forces across the highly mobile craniocervical junction.

Our case is different from those noted above in that there was no indication that the lumbar intrathecal catheter had fractured prior to the patient's presentation with
SAH. In each of the other cases of intracranial migration, ${ }^{7,11,12}$ it was known, or at least considered possible, that there was a free-floating piece of catheter in the thecal sac. In our case, no surveillance imaging had been undertaken to indicate that the catheter was fractured, the patient had no increase in chronic pain to indicate that the narcotic infusion system was malfunctioning, and there were no symptoms that could be referable to catheter fracture and migration, such as back pain or radiculopathy. There is no way to know how long the catheter tip had been located in the prepontine cistern. Perhaps the hemorrhage coincided with entry of the catheter tip into the prepontine cistern, or perhaps the catheter had been there for some time, and a small positional change resulted in the hemorrhage. Alternatively, the hemorrhage may have been completely independent of the migrated catheter and occurred secondary to the patient's anticoagulation.

Guidelines for the management of fractured lumbar intrathecal catheters in the spinal subarachnoid space are not established. The natural history of a free-floating catheter in the spinal subarachnoid space is not well known. The likelihood of a fractured catheter migrating and causing an adverse event such as intracranial SAH is presumably very low, and that would lead some surgeons to leave the fractured catheter in situ. However, as the discussed cases demonstrate, migration can occur, and it can result in adverse events. The two previous reports of migrated catheters causing intracranial $\mathrm{SAH},{ }^{11,12}$ in addition to this case, may cause surgeons to more strongly consider explanting these devices to prevent such an occurrence.

The optimal management of a migrated catheter in the basal cisterns in the setting of SAH is also not clear. There is certainly a risk to removing the catheter, as the hemorrhage may have caused it to scar in place, ${ }^{12}$ and withdrawing it may cause further hemorrhage. There is also risk to leaving the catheter in situ, as it may continue to shift position and cause further vascular injury. In our case, as in the cases reported by Guppy et al. ${ }^{7}$ and Nakaji et al., ${ }^{12}$ the catheter could be withdrawn easily without further hemorrhage.

\section{Conclusions}

Fracture and intracranial migration of lumbar intrathecal catheters is a rare complication. Subarachnoid hemorrhage is a possible sequela of migration. Although intracranial migration is rare, serious consideration should be given to removal of free-floating catheter fragments in the spinal subarachnoid space when they are discovered. Should a catheter migrate intracranially, with or without resultant $\mathrm{SAH}$, we suggest that explantation can be safe and should be performed in most cases.

\section{Acknowledgments}

We thank Shirley McCartney, $\mathrm{PhD}$, and Andy Rekito, MS, for editorial and illustrative assistance, respectively.

\section{References}

1. Albrecht E, Durrer A, Chédel D, Maeder P, Buchser E: Intraparenchymal migration of an intrathecal catheter three years after implantation. Pain 118:274-278, 2005 
2. Alleyne CH Jr, Shutter LA, Colohan AR: Cranial migration of a lumboperitoneal shunt catheter. South Med J 89:634636, 1996

3. Anthogalidis EI, Sure U, Hellwig D, Bertalanffy H: Intracranial dislocation of a lumbo-peritoneal shunt-catheter: case report and review of the literature. Clin Neurol Neurosurg 101:203-206, 1999

4. Fedorow CA, Moon MC, Mutch WA, Grocott HP: Lumbar cerebrospinal fluid drainage for thoracoabdominal aortic surgery: rationale and practical considerations for management. Anesth Analg 111:46-58, 2010

5. Flückiger B, Knecht H, Grossmann S, Felleiter P: Devicerelated complications of long-term intrathecal drug therapy via implanted pumps. Spinal Cord 46:639-643, 2008

6. Follett KA, Naumann CP: A prospective study of catheterrelated complications of intrathecal drug delivery systems. J Pain Symptom Manage 19:209-215, 2000

7. Guppy KH, Silverthorn JW, Akins PT: Subarachnoid hemorrhage due to retained lumbar drain. Case report. J Neurosurg Spine 15:641-644, 2011

8. Henry-Feugeas MC, Idy-Peretti I, Blanchet B, Hassine D, Zannoli G, Schouman-Claeys E: Temporal and spatial assessment of normal cerebrospinal fluid dynamics with MR imaging. Magn Reson Imaging 11:1107-1118, 1993

9. Kanai M, Kawano K, Uehara S: Upward migration of the L-P shunt catheter into the cranial base. Osaka City Med J 45:123-127, 1999

10. Ko WM, Ferrante FM: New onset lumbar radicular pain after implantation of an intrathecal drug delivery system: imaging catheter migration. Reg Anesth Pain Med 31:363367,2006
11. Maugans TA: Intracranial migration of a fractured intrathecal catheter from a baclofen pump system: case report and analysis of possible causes. Neurosurgery 66:319-322, 2010

12. Nakaji P, Consiglieri GD, Garrett MP, Bambakidis NC, Shetter AG: Cranial migration of a baclofen pump catheter associated with subarachnoid hemorrhage: case report. Neurosurgery 65:E1212-E1213, 2009

\section{Author Contributions}

Acquisition of data: all authors. Analysis and interpretation of data: Hnenny. Drafting the article: Hnenny, Sabry, Raskin, Liu, Roundy. Critically revising the article: Dogan, Hnenny. Reviewed submitted version of manuscript: all authors. Approved the final version of the manuscript on behalf of all authors: Dogan. Study supervision: Dogan.

\section{Supplemental Information}

\section{Current Affiliations}

Dr. Sabry: Department of Neurosurgery, Ain Shams University, Cairo, Egypt. Dr. Roundy: Neurosurgeon, Springfield, OR, and PeaceHealth Sacred Heart Medical Center at RiverBend, Springfield, OR.

\section{Correspondence}

Aclan Dogan, Department of Neurological Surgery, Oregon Health \& Science University, 3303 SW Bond Ave., Mail Code CH8N, Portland, OR 97239. email: dogana@ohsu.edu. 